\title{
THREE-DIMENSIONAL SIMULATION OF A SMALL-SCALE ELECTRODIALYSIS PROCESS FOR DOMESTIC USE
}

\author{
Noureddine Bensedira ${ }^{*}$, Mohammed-salah Aggoune ${ }^{1}$, Mabrouk Chabane ${ }^{1}$ and Said Drid ${ }^{2}$ \\ ${ }^{1}$ LEB Research Laboratory, Electrical engineering department, University of Batna 2, Algeria \\ E-mail: Noursbat@gmail.com, mohammedsalahaggoune@yahoo.com, \\ mabrouk_chabane@yahoo.com \\ ${ }^{2}$ LSP-IE Research Laboratory, Electrical engineering department, University of Batna 2, \\ Algeria \\ E-mail: s_drid@yahoo.fr, said.drid@univ-batna.dz \\ *corresponding author
}

\begin{abstract}
The magnetohydrodynamic (MHD) driving force and ionic transport characteristics of an electrolytic solution in a small-scale electrodialysis process are taken into account in this new approach. A three-dimensional modeling and simulation of coupling magnetohydrodynamicionic transport are carried out. The numerical values and the effects of applied voltage and magnetic induction on the fluid velocity and ionic concentration of species present in the solution are displayed graphically. The results show that a high decrease of ion concentration is achieved and suggest that the proposed model, which has no moving parts, can be practically implemented.
\end{abstract}

Keywords: Small-scale electrodialysis, MHD driving force, ionic transport, multiphysics coupling, Lorentz force, molar concentration, flow velocity field.

\section{Introduction}

Electrodialysis is a mass separation process in which electrically charged membranes and an electrical potential difference are used to separate ionic species present in an aqueous solution. This process is used today on a large industrial scale in the production of potable and industrial processed water as well as in the treatment of industrial effluents (Červenková et al. 2017, Akhter et al. 2018, Campione et al. 2020). A small-scale electrodialysis application is one of the most promising topics to expand because these technologies will contribute to improving the living conditions of people especially in deserted and rural areas. Much work with small-scale units, especially for removal and recovery of some elements found in wastewater, has been achieved during the last two decades (Schoeman 2009, Matamoros 2009, Mahmoud 2012).

With regard to saltwater desalination, Omar Chaalal and al designed a small-scale electrodialysis system in 2015 consisting of six compartments and conducted a series of experiments on the transport of sodium $\left(\mathrm{Na}^{+}\right)$and chloride ions $\left(\mathrm{Cl}^{-}\right)$. The effects of electric current and voltage on the Salinity of water were investigated. The results obtained confirm that this device has the 
potential for domestic use in isolated houses where potable water supply is not available. This was achieved when two of the small-scale electro-dialysers were placed in series and the solutions from the respective compartments were mixed (Chaalal et al. 2015).

In 2017, an alternative solution to the reverse osmosis (RO) method for water desalination in homes was designed and experimentally shown by Kishor G. Nayar and al. The results indicate that it is feasible to design a small-scale electrodialysis system that can process groundwater while providing a higher water recovery than RO products. Furthermore, it was shown that the cost of the proposed system was estimated to be within the price range of existing in-home RO products, with potential reductions in overall costs (Nayar et al. 2015, 2017).

Electrodialysis process is a complex operation where the flow of fluid to be treated is usually ensured by external pumps making the system a bulky device; these systems cannot be easily miniaturized for practical use in the laboratory, at home or for mobile purpose (Sistat et al. 2005, Červenková et al. 2017). In an experimental study conducted by J. J. Schoeman, the feasibility of using small techniques (reverse osmosis, ion exchange and electrodialysis) to remove nitrates and nitrogen from water was considered for use at the clinic level in Limpopo Province. He noted that the electrolysis process is suitable for achieving the purpose, but it is more complicated and difficult to operate in rural areas (Schoeman 2009). In 2005, a new design for large-scale water desalination, where the fluid flow in the electrodialyser system is generated by a magnetohydrodynamic (MHD) effect, was proposed by Philippe Sistat and al, (Sistat et al. 2005).

In 2005, Ortiz et al. developed a mathematical model for desalination of brackish water through controlled potential electrodialysis. The application of this model allows predicting system behavior, calculating electrical energy consumption and calculating the time required for successful desalination (Ortiz et al. 2005). Following this advancement in the modeling process, the improvement and characterization of homegrown electrodialysis systems became available in a way that was not previously possible. M. Qin et al. proposed a model that treats the movement of a solution in a channel and provides detailed information on ion concentration (Qin et al. 2009). In a study conducted by F.S. Rohman et al., the authors presented a model able to separate the ionic chemicals from non-ionic chemicals in the process of waste streams (Rohman et al. 2010). R. Kwak et al. performed a scaling analysis, which allows one to predict ion concentrations and fluid flows (Kwak et al. 2013). R. Enciso et al. carried out a simulation of an electrodialysis system under the laminar flow condition and the emphasis was on the mass transfer rate and its influence on the concentration polarization (Enciso et al. 2017).

In this article, we present a new approach of a small-scale electrodialysis for domestic and mobile water desalination where the flow of saltwater is generated by MHD driving force, which makes the process practical and less cumbersome as it uses no external pumps. This proposed model is energy efficient as it uses only an external DC source to supply the necessary current needed to pump the solution through the system. Three-Dimensional modeling and simulation of coupling Magnetohydrodynamic-Ionic transport in the solution are carried out.

\section{Problem description}

\subsection{Electrodialysis (ED) system}

The term "electrodialysis" refers to the transfer of ions through membranes under the effect of an electric field. Electrodialysis uses ion exchange membranes alternately arranged: cation exchange membranes (CEM) and anion exchange membranes (AEM) separated by a separator frame that ensures the hydrodynamics of the system and its tightness. A schematic view of an electrodialysis cell is shown in Fig. 1. 
Under the effect of an electric field, the cations migrate to the cathode: they leave the compartments D by crossing a cationic membrane CEM, but they cannot leave the compartments $\mathrm{B}$ because they are blocked by an anionic membrane AEM. As a result, the D compartments are depleted in salts and are called "desalination or diluted compartments". Compartments B are enriched in salts, called "brine or concentrated compartments".

Current distribution is provided by a pair of electrolyte-fed electrodes separated from the diluted or concentrated circuits by an end membrane. All of these elements are held between two plates under a high clamping pressure, thus constituting a reactor or stack. The unitary pattern is named cell. The same reactor can contain several hundred cells (Oztekin et al. 2016, Campione et al. 2020).

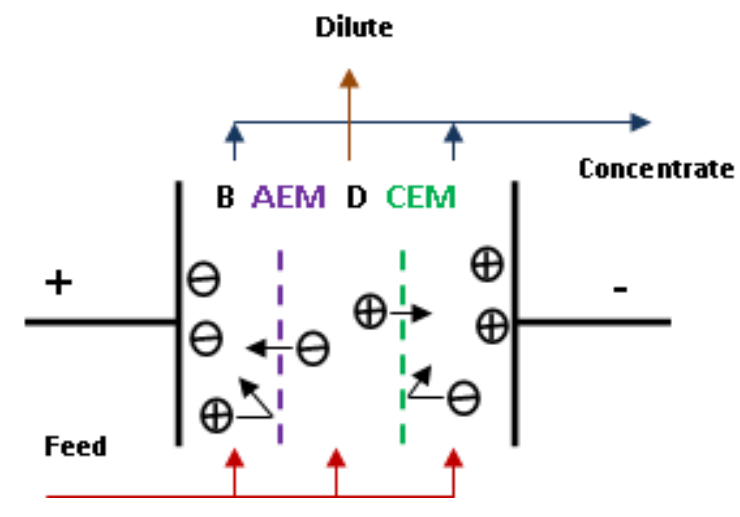

Fig. 1. A schematic view of an electrodialysis cell

Among the factors that strongly influence the efficiency and performance of the ED process are the properties of the membranes used, such as electrical conductivity, exchange capacity and thickness, whether they are anions (AEM) or cations (CEM) exchange membranes. In regards to the electrical membrane resistance of ion-exchange membranes (or reciprocal) electrical conductivity is important for the energy requirement of ED processes. In most practical cases the membrane thickness and electrical membrane resistance are much lower than the thickness and electrical resistance of the dilute solution (Tanaka 2009, Chehayeb et al. 2017).

\subsection{MHD Pumping Theory}

Magnetohydrodynamics (MHD) is the theory of the interaction of electrically conducting fluids and electromagnetic field. Application arises in astronomy and geophysics as well as in connection with numerous engineering problems, such as liquid metal cooling of nuclear reactors, electromagnetic casting of metals, MHD power generation and propulsion. One of the most important applications of this effect is pumping where the main factor responsible for the flow of the fluid is the Lorentz force; its expression is given by the following equation (Al-Habahbeh et al. 2016, Reddy et al. 2019, Rana et al. 2019):

$$
\vec{F}=\vec{J} \times \vec{B}
$$

Where $\vec{B}$ and $\vec{J}$ are the magnetic induction and the current vector density, respectively.

Figure 2 represents the basic design concept of an MHD pump; its principle is to apply an electrical current across a channel filled with electrically conducting liquid subject to a magnetic field orthogonal to the currents via a permanent magnet. 


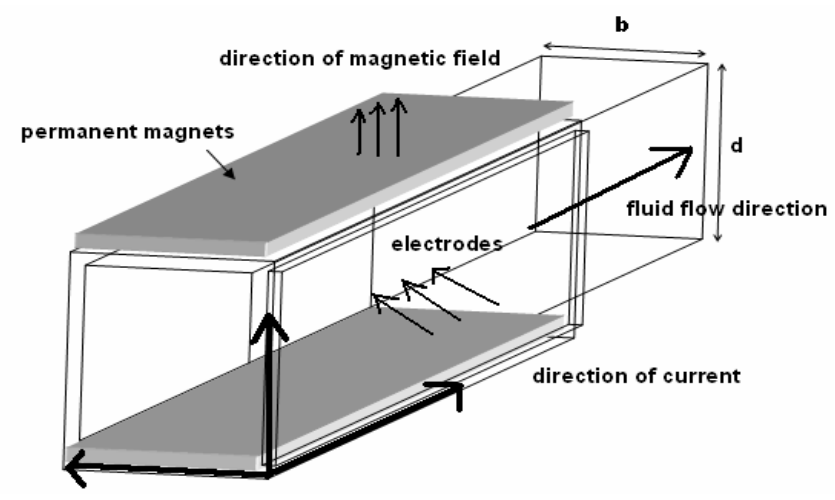

Fig. 2. Schematic design concept of an MHD pump

\subsection{Design of proposed process}

In Fig. 3 a small-scale electrodialysis system was proposed for domestic use. It is composed of a permanent magnet added to an electrodialysis device. As a consequence of the combination of electric current and magnetic field, the Lorentz force acts on the fluid in all compartments. As each compartment is separated by an alternance of cation and anion exchange membrane, there are concentrate and diluate compartments. This system represents a simple portable design, having no moving parts.

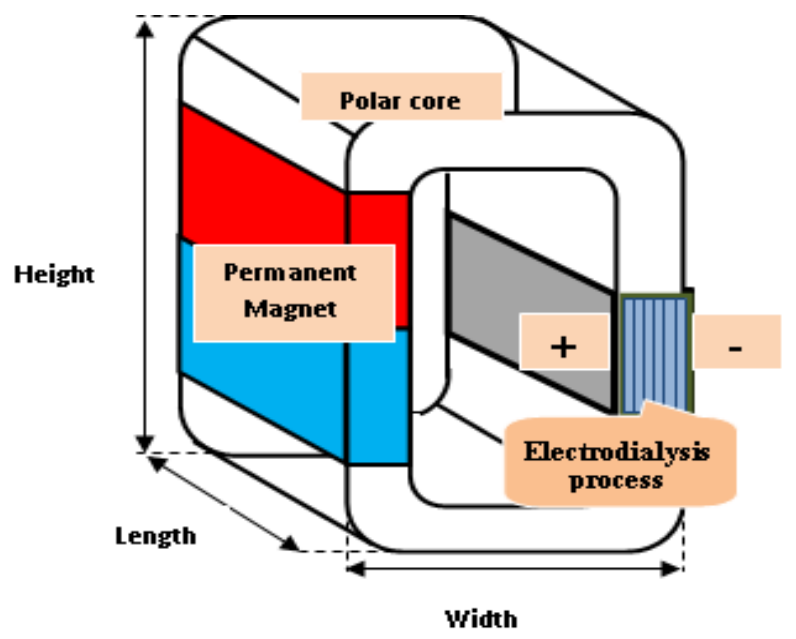

Fig. 3. Process configuration

The simulation is carried on an individual cell prototype of $(20 \times 20 \times 5) \mathrm{mm}$ in length, height and width respectively; it consists of three consecutive compartments of $(5 \times 0.5) \mathrm{mm}$ in height and width of each compartment respectively. When a DC potential is applied between two electrodes, positively charged cations move toward the cathode, pass through the negatively charged cation exchange membrane and retained by the positively charged anion exchange membrane. On the other hand, negatively charged anions move toward the anode, pass through the positively charged anion exchange membrane and retained by the negatively charged cation exchange membrane. At the end, the result will be a diluted compartment between two other 
concentrates. At first, doing the simulation to view profiles of fluid velocity and concentration of species present in the solution by preliminary values of applied voltage and magnetic induction ( $V_{a}=0.2$ volts and $B_{r}=0.5 T$ ); in the second step, we study the effects of applied voltage and magnetic induction on the fluid velocity and ionic concentration.

The seawater has an average salinity of $35 \mathrm{~g} / \mathrm{L}$ and more than $85 \%$ of the ions present in it are the $\mathrm{Na}^{+}$and $\mathrm{Cl}^{-}$ions. In this work, we consider the salt solution as a $\mathrm{NaCl}$ solution with an initial concentration that is equivalent to the average ratio of ions of $\mathrm{Na}^{+}$and $\mathrm{Cl}^{-}$present in seawater: 29.25g/L (500mol/L) (Mathon 2008, WHO. 2011).

The concentration is among the factors affecting the solution properties and to approach the behavior of these properties, the variation of electrical conductivity and density as a function of concentration of species present in the solution are taken into account in this new research; Their values are calculated respectively using the following equations (Snyder et al. 2003, Mathon 2008):

$$
\begin{gathered}
\sigma=\lambda_{1} \times c_{1}+\lambda_{2} \times c_{2} \\
\rho=\rho_{0}+M_{1} \times c_{1}+M_{2} \times c_{2}
\end{gathered}
$$

The quantities $\lambda_{i}, M_{i}$ and $c_{i}$ are the molar conductivity, the molar mass and the molar concentration of specie (i) where the index i can take one of the values 1 and 2 for $\mathrm{Cl}^{-}$and $\mathrm{Na}^{+}$ respectively.

\section{Mathematical models}

The modeling of the system requires the use of three physical models: magnetostatic, hydrodynamic and ionic transport. The three models must be used and coupled to simulate the system performance. The equations used to describe the three physical phenomena are presented:

\subsection{Magnetostatic model}

Maxwell's equations globally describe all electromagnetic phenomena. The constituent laws of the material, which are characteristic of each medium, must be added to the Maxwell equations to treat any particular electromagnetic problem. There are a large number of formulations of Maxwell's equations. In this work we have chosen the $(\vec{A}-V)$ formulation that allows the reduction of the number of unknowns (Bennecib et al. 2009, Kadid et al. 2011, Naceur et al. 2016, Hasan et al. 2017):

$$
\begin{gathered}
\vec{\nabla} \times\left(\mu_{0}^{-1} \mu_{\mathrm{r}}^{-1}\left(\vec{\nabla} \times \overrightarrow{\mathrm{A}}-\overrightarrow{\mathrm{B}}_{\mathrm{r}}\right)\right)-\sigma(\overrightarrow{\mathrm{u}} \times(\vec{\nabla} \times \overrightarrow{\mathrm{A}}))=-\sigma \vec{\nabla} V \\
\vec{\nabla} \cdot \vec{A}=0
\end{gathered}
$$

Where $\mu_{0}$ is the magnetic permeability of the vacuum, $\mu_{r}$ is the relative magnetic permeability, $\vec{A}$ is the magnetic vector potential, $\vec{B}_{r}$ is the residual induction, $\sigma$ is the electrical conductivity, $\vec{u}$ is the Fluid velocity and $\vec{A}^{*}$ the electric scalar potential. 


\subsection{Hydrodynamic model}

The equations governing the flow of the incompressible laminar fluid with constant properties are the conservation equations of the momentum and mass. They form the system to solve (Bennecib et al. 2009, Kadid et al. 2011, Naceur et al. 2016, Hasan et al. 2017):

$$
\begin{gathered}
\frac{(\partial \vec{u})}{\partial t}+(\vec{u} \cdot \vec{\nabla}) \vec{u}=-\frac{1}{\rho} \vec{\nabla} P+\vartheta \Delta \vec{u}+\frac{1}{\rho} \vec{F} \\
\vec{\nabla} \cdot \vec{u}=0
\end{gathered}
$$

Where $\vec{u}$ is the fluid velocity, $\rho$ its density, $P$ is the fluid pressure and $\vartheta$ is the kinematic viscosity of the fluid.

The term $\vec{F}$ represents the Lorentz force; according to equation (3); this force is the driving force applied to the fluid which can create the flow of the electrolyte solution into the compartments even in absence of external pressure difference between the inlet and outlet of the cell (Hasan et al. 2017).

\subsection{Ionic transport model}

In solutions, the ions move under the effect of: the concentration gradient (diffusion), the potential gradient (migration), and the flow of the solution (convection). The Nernst-Plank equation reflects the fact that the total molar ion flux of a species (i) is written as follows (Mathon 2008, Knust et al. 2014):

$$
\vec{N}_{i}=-D_{i} \vec{\nabla} c_{i}-z_{i} u_{m i} F_{f} c_{i} \vec{\nabla} V+c_{i} \vec{u}
$$

Where $\vec{N}_{i}$ is the molar ionic flux of the species (i), $D_{i}$ its coefficient of diffusion, $z_{i}$ the charge, $u_{m i}$ the ionic mobility, $\mathrm{F}$ is the Faraday constant, $\mathrm{V}$ is the electric potential, $c_{i}$ is the molar concentration and $\vec{u}$ is the fluid velocity.

We add to the conservation of the total mass contained in a fluid the conservation of the species present in the fluid and the evolution of the local concentration of the species (i) is then given by the following equation (Mathon 2008, Knust et al. 2014):

$$
\frac{\partial c_{i}}{\partial t}+\vec{\nabla} \cdot \vec{N}_{i}=0
$$

The spatio-temporal variations of the ionic exchange of the species present in the fluid are governed by the following equation $[20,26]$ :

$$
\frac{\partial c_{i}}{\partial t}+\vec{\nabla} \cdot\left(-D_{i} \vec{\nabla} c_{i}-z_{i} u_{m i} F_{f} c_{i} \vec{\nabla} V\right)=-\vec{u} \cdot \vec{\nabla} c_{i}
$$

In this article, the work is based mainly on the study of the behavior of the hydrodynamic and ionic exchanges of the solution at the level of the diluted compartment (D). The ion exchange of $\mathrm{Na}^{+}$between the dilated compartment and the compartment adjacent to the negative electrode will be limited because the passage of $\mathrm{Na}^{+}$through the AEM membrane is not allowed, while $\mathrm{Cl}^{-}$is not allowed to pass the CEM membrane. We will limit its transfer between the dilated compartment and the other compartments adjacent to the positive electrode and we considered that the cations $\mathrm{Na}^{+}$in compartments adjacent to the positive electrode and the anions $\mathrm{Cl}^{-}$in 
the compartment adjacent to the negative electrode are influenced only by the convective term ( $\left.\frac{\partial c_{i}}{\partial t}=-\vec{u} \cdot \vec{\nabla} c_{i}\right)$.

In this work, 3D modeling and simulation are carried out using a finite element method with Comsol 3.5 software. Taking into account all appropriate boundary conditions to solve the system of equations, Comsol proposes a set of solvers able to simulate the magnetostatic, hydrodynamic and ionic transport aspects with their couplings; here, the numerical resolution is carried out by the direct solver: SPOOLES (SParse Object Oriented Linear Equations Solver).

As for the parameters used in this work, a summary of the characteristics is presented in Table 1. below:

\begin{tabular}{|c|c|}
\hline Parameter & Values \\
\hline Relative magnetic permeability of solution & 1 \\
\hline $\begin{array}{c}\text { Relative magnetic permeability of polar } \\
\text { core }\end{array}$ & 5000 \\
\hline Dynamic viscosity & $0.996 \times 10^{-2} \mathrm{Pa.s}$ \\
\hline Coefficient of diffusion & $D_{1}=2.03 \times 10^{-9} \mathrm{~m}^{2} / \mathrm{s}$ \\
& $D_{2}=1.33 \times 10^{-9} \mathrm{~m}^{2} / \mathrm{s}$ \\
\hline Ionic mobility & $u_{m 1}=8.193506 \times 10^{-9} \mathrm{~s} . \mathrm{mol} / \mathrm{Kg}$ \\
& $u_{m 2}=5.368159 \times 10^{-9} \mathrm{~s} . \mathrm{mol} / \mathrm{Kg}$ \\
\hline Molar conductivity & $\lambda_{1}=7.63 \times 10^{-3} \mathrm{~S} . \mathrm{m}^{2} / \mathrm{mol}$ \\
& $\lambda_{2}=5.01 \times 10^{-3} \mathrm{~S} . \mathrm{m}^{2} / \mathrm{mol}$ \\
\hline Molar mass & $M_{1}=35.5 \mathrm{~g} / \mathrm{mol} \mathrm{M} \mathrm{M}_{2}=23 \mathrm{~g} / \mathrm{mol}$ \\
\hline Water density & $\rho_{0}=998 \mathrm{Kg} / \mathrm{m}^{3}$ \\
\hline
\end{tabular}

Table 1. Different values of system properties (Mathon 2008, Knust et al. 2014)

\section{Simulations and results}

The interaction between the magnetic flux density and induced current density applied on the electrodialysis cell yields the Lorentz force, which puts the solution into motion. These parameters are shown in the simulation results presented in Figs. 4, 5 and 6. 


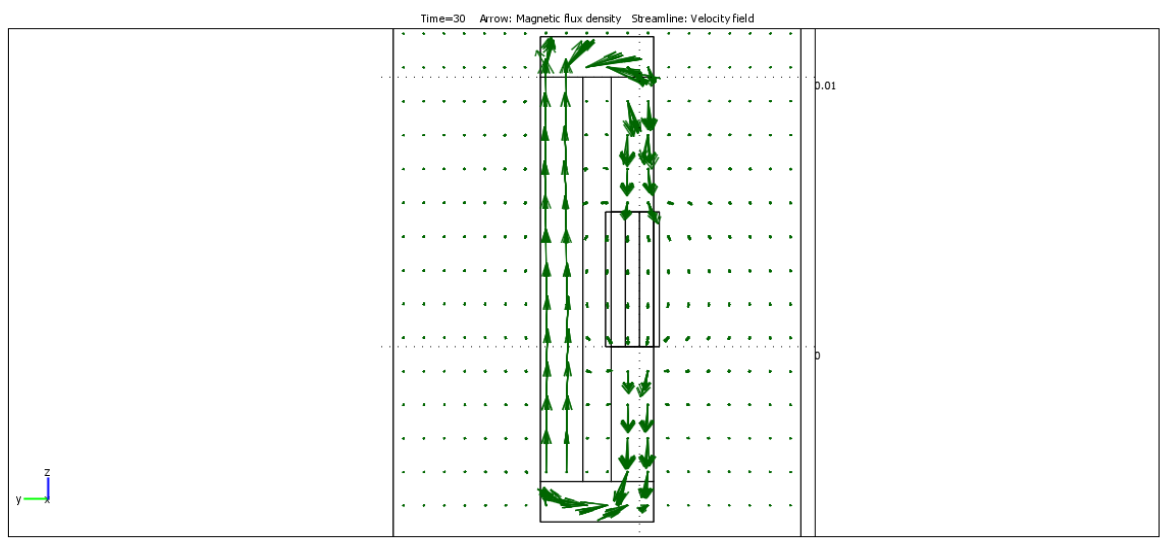

Fig. 4. Magnetic flux density distribution

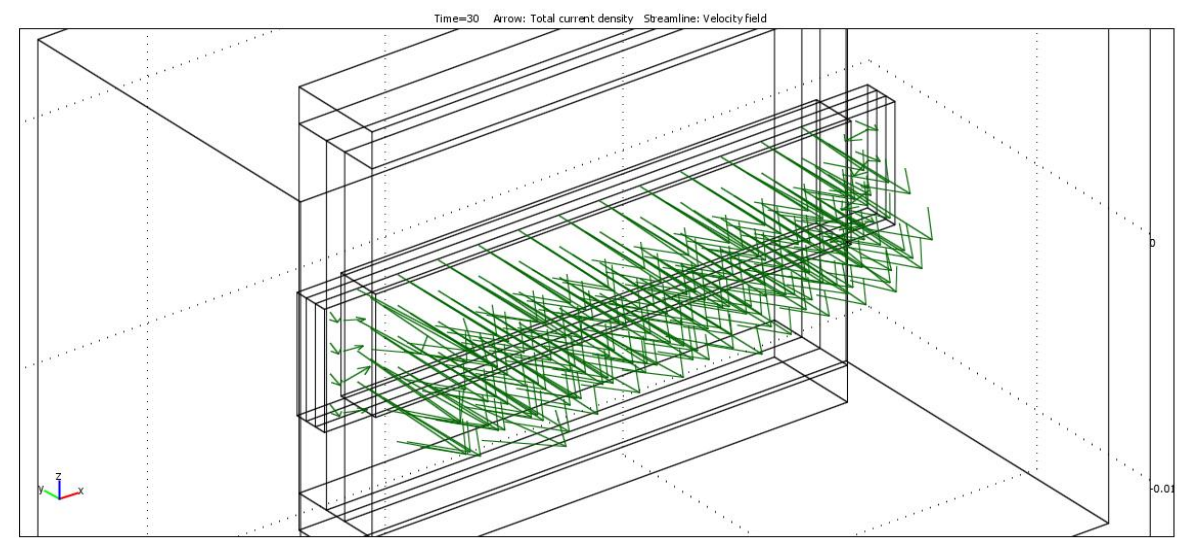

Fig. 5. Induced current density distribution

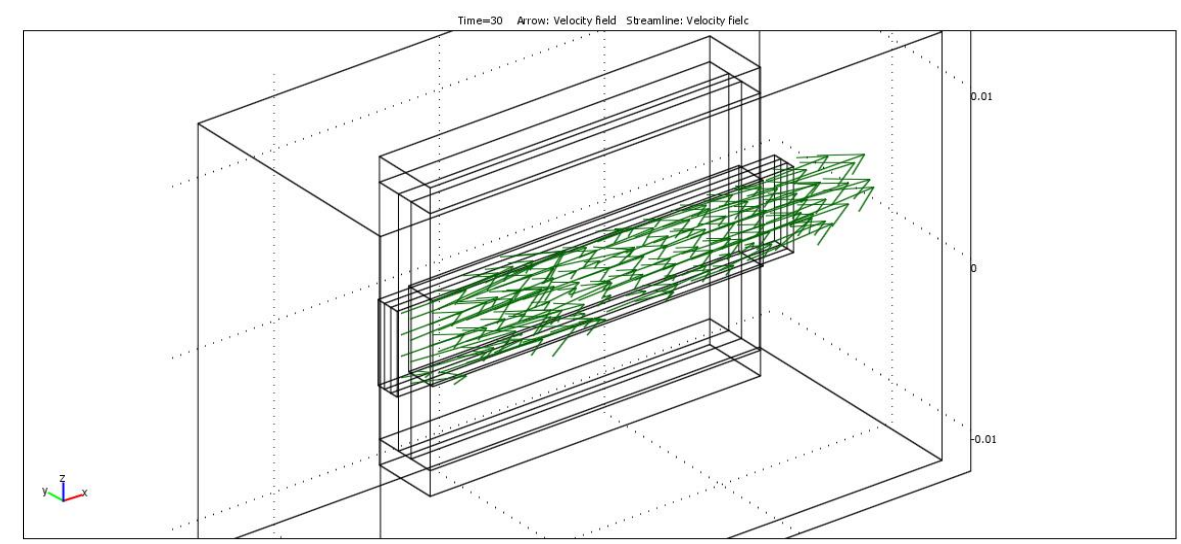

Fig. 6. Flow velocity field 
Figure 7 shows the flow velocity profile at the cell outlet at steady state for $(t=30 \mathrm{~s})$, this profile is parabolic; the maximum values of this velocity are located at the centers of the compartments. These values gradually decrease to take minimum values on the walls of the compartments. In the outlet of the diluted compartment; the temporal evolution visualization of velocity is shown in Fig. 8; we note that this velocity passes by a transient state and then stabilizes at a value of: $u=1.5 \mathrm{~mm} / \mathrm{s}$.

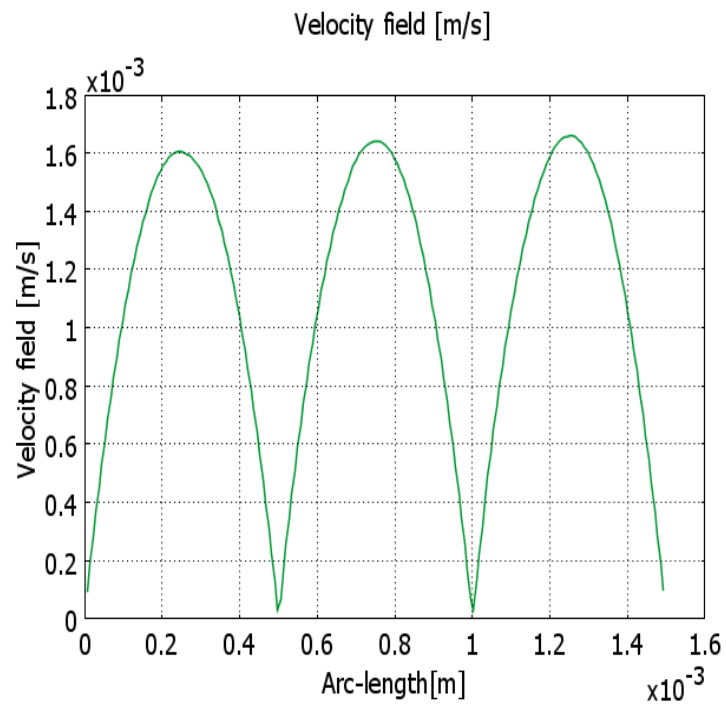

Fig. 7. Flow velocity profile at the cell center

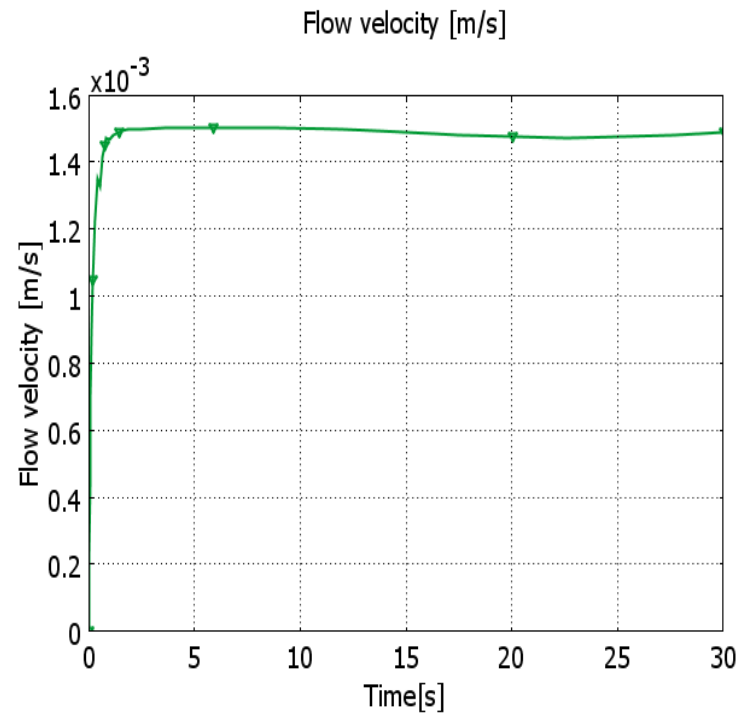

Fig. 8. Evolution of diluted fluid velocity at exit

In order to visualize the cell's ability to be desalinated, the horizontal profiles of the concentrations of species present in the solution at the inlet, the middle and at the outlet of the 
dilute compartment are shown in Figs. 9 and 10 at $(\mathrm{t}=30 \mathrm{~s})$. In this later case, we observe that the concentration of $\mathrm{Cl}^{-}\left(c_{1}\right)$ is minimal $\left(c_{1}=150 \mathrm{~mol} / \mathrm{m}^{3}\right)$ on the left wall (CEM); this value increases gradually to reach a maximum value $\left(c_{1}=425 \mathrm{~mol} / \mathrm{m}^{3}\right)$ on the right wall (AEM), whereas we notice that the concentration of $\mathrm{Na}^{+}\left(c_{2}\right)$ is minimal $\left(c_{2}=175 \mathrm{~mol} / \mathrm{m}^{3}\right)$ on the right wall (AEM) then it gradually increases to a maximum value $\left(c_{2}=465 \mathrm{~mol} / \mathrm{m}^{3}\right)$ on the left wall (MEC). It is clear that the average values of these concentrations are lower than the initial concentration $\left(c_{0}=500 \mathrm{~mol} / \mathrm{m}^{3}\right)$.

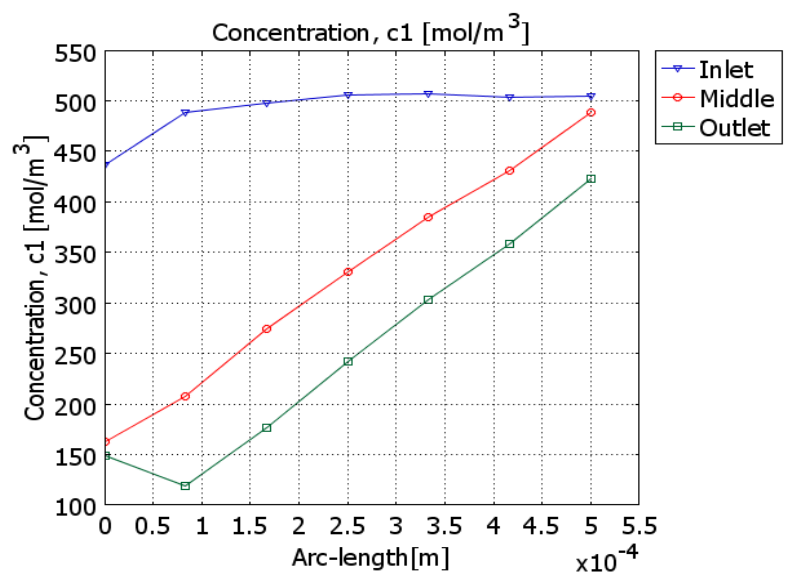

Fig. 9. Horizontal profile of concentration of $\mathrm{Cl}^{-}$at the diluted compartment

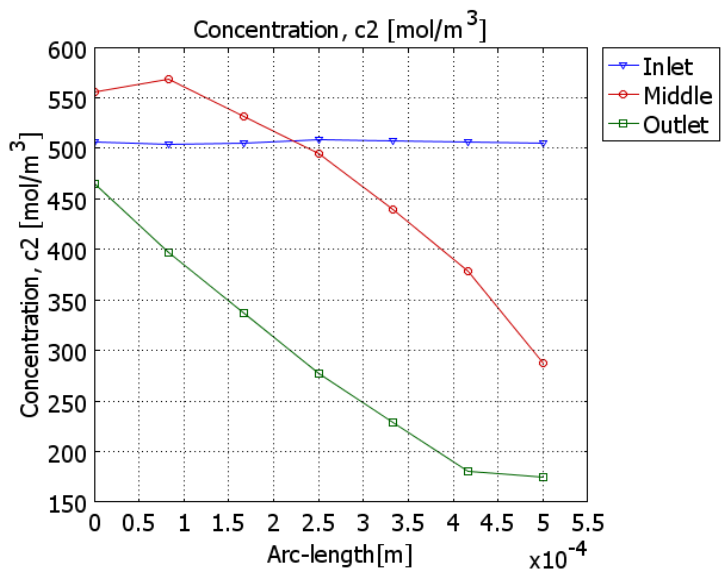

Fig. 10. Horizontal profile of concentration of $\mathrm{Na}^{+}$at the diluted compartment

In Fig. 11, we present the ionic concentration along the diluted compartment. The concentration profiles of species present in the solution show that the concentration values are maximal and roughly equal to the initial concentration $\left(c_{0}=500 \mathrm{~mol} / \mathrm{m}^{3}\right)$ at the inlet of compartments, then they gradually decrease to minimal values at the outlet. This process can reduce the ion concentration by $60 \%$ for $\mathrm{Cl}^{-}$and $5 \% \%$ for $\mathrm{Na}^{+}$. 


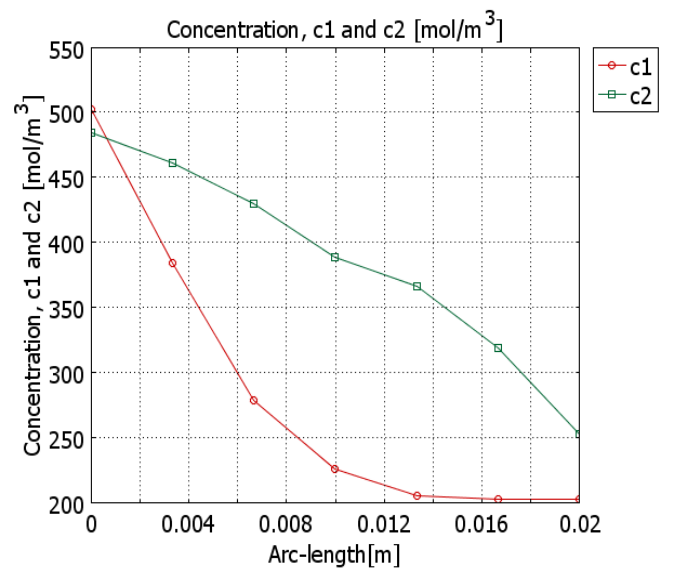

Fig. 11. Ionic concentration along the diluted compartment

The results obtained in the previous paragraph indicate that the main parameters of our model are able to decrease the ionic concentration according to theory but their final values are still high. To go further, we investigated the influence of the variations in the applied voltage and induction on the overall performance of the proposed system. At first, the simulation was repeated several times in which the applied voltage was varied from 0 volts to 3 volts and the induction increased from $0.15 \mathrm{~T}$ to $0.45 \mathrm{~T}$; in the second step, the Matlab computer tool was used to plot the evolution of the velocity and the concentration as a function of the applied voltage.

The velocity tends to proportionality increase with both the magnetic field and the applied voltage due to an increase in the Lorentz force. As for the concentration, the simulation shows that the best results are obtained for higher applied voltages for any given magnetic field.

According to the results obtained, it was found that there is a net improvement in both the flow velocity and especially the reduction of solution concentration, which decreased to $0.57 \mathrm{~g} / \mathrm{L}$ with a velocity of $3.75 \mathrm{~mm} / \mathrm{s}$ for an induction of $0.15 \mathrm{~T}$ and an applied voltage of 3 volts as shown in Figs. 13, 14 and Table 2.

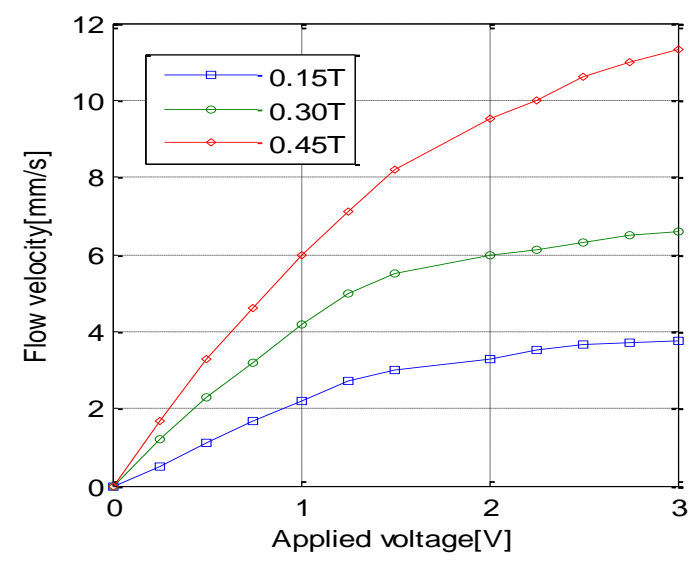

Fig. 12. Effect of applied voltage and induction on flow velocity 


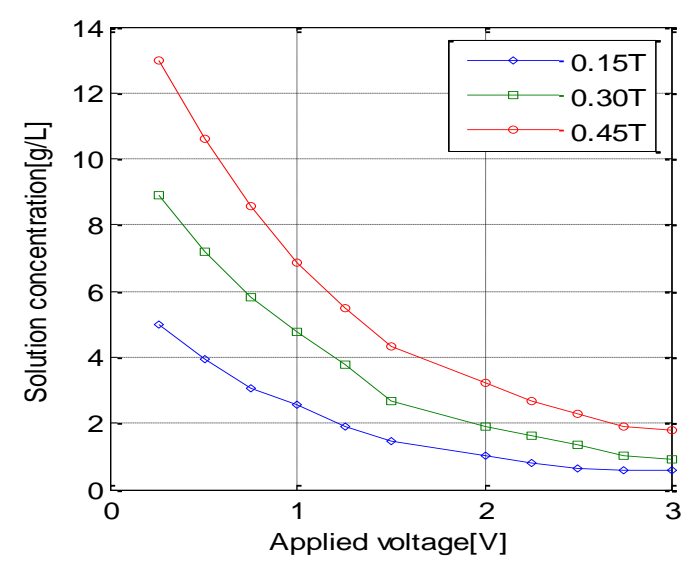

Fig. 13. Effect of applied voltage and induction on solution concentration

\begin{tabular}{|c|c|c|c|}
\hline Induction [T] & Voltage [V] & Flow velocity $[\mathrm{mm} / \mathrm{s}]$ & concentration, $[g / L]$ \\
\hline \multirow{3}{*}{0.15} & 1 & 2.20 & 2.57 \\
& 2 & 3.30 & 1.00 \\
& 3 & 3.75 & 0.57 \\
\hline \multirow{3}{*}{0.30} & 1 & 4.20 & 4.79 \\
& 2 & 6.00 & 1.90 \\
& 3 & 6.60 & 0.90 \\
\hline \multirow{3}{*}{0.45} & 1 & 6.00 & 6.84 \\
& 2 & 9.50 & 3.20 \\
& 3 & 11.3 & 1.78 \\
\hline
\end{tabular}

Table 2. Effect of applied voltage and induction on solution velocity and concentration

Finally, from an industrial point of view, the number and dimensions of the cells must be adapted mainly to the source of energy available (solar panels, battery ...).

\section{Conclusion}

In the present paper, a small-scale electrodialysis process for domestic use was proposed and a three-dimensional simulation of a magnetohydrodynamic-ionic transport coupling was presented. The model makes it possible to predict the concentration of dilute stream or separation percent of the cell at different operating conditions; various characteristics, mainly the effect of the voltages and the magnetic induction in the flow velocity and the ionic concentration of $\mathrm{Cl}^{-}$and $\mathrm{Na}^{+}$are investigated. The results predict a flow of solution accompanied by a decrease of the concentration of both ions at the outlet, which makes this application a practical and less cumbersome process as it is a simple design that uses no external pumps and has no moving parts.

\section{References}

Akhter, M., Habib, G., \& Qama, S. U. (2018). Application of electrodialysis in waste water treatment and impact of fouling on process performance, J Membr Sci Technol, 8(2), 1-8. 
Al-Habahbeh, O. M., Al-Saqqa, M., Safi, M., \& Khater, T. A. (2016). Review of magnetohydrodynamic pump applications, Alexandria Engineering Journal, 55(2), 13471358 .

Bennecib, N., Drid, S., \& Abdessemed, R. (2009). Numerical investigation of flow in a new DC pump MHD, Journal of applied fluid mechanics, 2(2), 23-28.

Campione, A., Cipollina, A., Toet, E., Gurreri, L., Bogle, I. D. L., \& Micale, G. (2020). Water desalination by capacitive electrodialysis: Experiments and modelling, Desalination, 473, 114150.

Červenková, M., Chromíková, J., Heviánková, S., \& Wranová, Z. (2017). The application of electrodialysis for the recovery of phosphorus from wastewater sludge liquid discharge, E\&ES, 92(1), 012007.

Chaalal, O., \& Hossain, M. M. (2015). A small-scale membrane electro-dialyser for domestic use, Membrane Water Treatment, 6(1), 43-52.

Chehayeb, K. M., Farhat, D. M., \& Nayar, K. G. (2017). Optimal design and operation of electrodialysis for brackish-water desalination and for high-salinity brine concentration, Desalination, 420, 167-182.

Enciso, R., Delgadillo, J. A., Domínguez, O., \& Rodríguez-Torres, I. (2017). Analysis and validation of the hydrodynamics of an electrodialysis cell using computational fluid dynamics. Desalination, 408, 127-132.

Hasan, M. I., Ali, A. J. F., \& Tufah, R. S. (2017). Numerical study of the effect of channel geometry on the performance of Magnetohydrodynamic micro pump, Engineering science and technology, an international journal, 20(3), 982-989.

Kadid, F. Z., Drid, S., \& Abdessemed, R. (2011). Simulation of magnetohydrodynamic and thermal coupling in the linear induction MHD pump, Journal of Applied Fluid Mechanics, 4(1), 51-57.

Knust, K. N., Hlushkou, D., Tallarek, U., \& Crooks, R. M. (2014). Electrochemical desalination for a sustainable water future, ChemElectroChem, 1(5), 850-857.

Kwak, R., Lim, K. M., \& Han, J. (2013). Shear flow of an electrically charged fluid by ion concentration polarization: Scaling laws for electroconvective vortices. Physical review letters, 110(11), 114501.

Mahmoud, A., \& Hoadley, A. F. (2012). An evaluation of a hybrid ion exchange electrodialysis process in the recovery of heavy metals from simulated dilute industrial wastewater, Water research, 46(10), 3364-3376.

Matamoros, V., Arias, C., Brix, H., \& Bayona, J. M. (2009). Preliminary screening of small-scale domestic wastewater treatment systems for removal of pharmaceutical and personal care products. Water research, 43(1), 55-62.

Mathon, P. (2008). Influences des forces électromagnétiques sur les processus électrochimiquesApplication à la propulsion MHD, Doctoral dissertation presented to the Grenoble polytechnic institute, France, 115-126.

Naceur, S., Kadid, F. Z., \& Abdessemed, R. (2016). The study of the electroconductive liquids flow in a conduction magnetohydrodynamic pump, Transactions on Electrical and Electronic Materials, 17(5), 252-256.

Nayar, K. G., Sundararaman, P., Schacherl, J. D., O’Connor, C. L., Heath, M. L., Orozco Gabriel, M., ... \& Winter, A. G. (2015, August). Feasibility study of an electrodialysis system for inhome water desalination and purification in urban India. In International Design Engineering Technical Conferences and Computers and Information in Engineering Conference (Vol. 57076, p. V02AT03A046). American Society of Mechanical Engineers.

Nayar, K. G., Sundararaman, P., O'Connor, C. L., Schacherl, J. D., Heath, M. L., Gabriel, M. O., ... \& Winter, A. G. (2017). Feasibility study of an electrodialysis system for in-home water desalination in urban India, Development Engineering, 2, 38-46. 
Ortiz, J. M., Sotoca, J. A., Expósito, E., Gallud, F., Garcia-Garcia, V., Montiel, V., \& Aldaz, A. (2005). Brackish water desalination by electrodialysis: batch recirculation operation modeling. Journal of membrane science, 252(1-2), 65-75.

Oztekin, E., \& Altin, S. (2016). Wastewater treatment by electrodialysis system and fouling problems, Turkish Online Journal of Science \& Technology, 6(1). 91-99.

Qin, M., \& Bau, H. H. (2009). Magneto-hydrodynamic flow in electrolyte solutions. In Comsol conference.

Reddy, B. P., \& Peter, J. (2019). Effects of Chemical Reaction on MHD Flow Past an Impulsively Started Infinite Vertical Plate with Variable Temperature and Mass Diffusion in the Presence of Hall Current. Journal of the Serbian Society for Computational Mechanics, 13(1), 92-108.

Rana, G. C., Gautam, P. K., \& Saxena, H. (2019). Electrohydrodynamic thermal instability in a Walters (model B') rotating nanofluid saturating a porous medium, Journal of the Serbian Society for Computational Mechanics, 13(2), 19-35.

Rohman, F. S., Othman, M. R., \& Aziz, N. (2010). Modeling of batch electrodialysis for hydrochloric acid recovery. Chemical Engineering Journal, 162(2), 466-479.

Schoeman, J. J. (2009). Nitrate-nitrogen removal with small-scale reverse osmosis, electrodialysis and ion-exchange units in rural areas. Water SA, 35(5), 721-728.

Sistat, P., Huguet, P., \& Nikonenko, V. (2005). Magnetohydrodynamic flow in electrodialyser channel, 207th ECS Meeting, Quebec City (Canada).

Snyder, K. A., Feng, X., Keen, B. D., \& Mason, T. O. (2003). Estimating the electrical conductivity of cement paste pore solutions from $\mathrm{OH}-, \mathrm{K}+$ and $\mathrm{Na}+$ concentrations, Cement and Concrete Research, 33(6), 793-798.

Tanaka, Y. (2009). A computer simulation of continuous ion exchange membrane electrodialysis for desalination of saline water, Desalination, 249(2), 809-821.

WHO (2011). Guidelines for Drinking-water Quality. Fourth edition, 20 Avenue Appia, 1211 Geneva 27, Switzerland. 